\title{
Chronische Hepatitis C
} Nierenfunktion wichtig für die Therapieentscheidung

Grundsätzlich spielt die Nierenfunktion eine wichtige Rolle für zahlreiche Pharmakotherapien, vor allem, wenn Medikamente überwiegend renal ausgeschieden werden. Dies trifft auch für die antivirale Therapie der Hepatitis-C-Virus(HCV)Infektion zu. Hier besitzt dieser Aspekt sogar besondere Bedeutung, weil die HCV-Infektion selbst das Risiko für die Entstehung und das Fortschreiten einer Niereninsuffizienz erhöht [1]. Die Nierenfunktion sollte deshalb vor Beginn einer antiviralen Therapie analysiert werden. Das Therapieregime Viekirax ${ }^{\circledR} \pm$ Exviera $^{\circledR}$ ist laut Leitlinie der Deutschen Gesellschaft für Gastroenterologie, Verdauungs- und Stoffwechselkrankheiten (DGVS) als einzige Behandlungsoption auch für Patienten mit schwerer Nierenfunktionsstörung möglich [2].

Eine Anomalie der Nierenstruktur oder -funktion, die länger als 3 Monate besteht und die Gesundheit beeinträchtigt, wird als chronische Nierenerkrankung bezeichnet. Funktioneller Ausdruck dieser Veränderungen ist eine Abnahme der glomerulären Filtrationsrate (GFR). Eine GFR von 60 bis $89 \mathrm{ml} / \mathrm{min} / 1,73 \mathrm{~m}^{2}$ gilt als leichte Einschränkung der Nierenfunktion, eine GFR $<15$ $\mathrm{ml} / \mathrm{min} / 1,73 \mathrm{~m}^{2}$ als Nierenversagen [3].

\section{Hohe Prävalenz der Nieren-}

\section{insuffizienz in Deutschland}

In Deutschland findet sich eine im europäischen Vergleich relativ hohe Prävalenz der Niereninsuffizienz bei 45 - bis 74-Jährigen von 25,6\% [4]. Patienten mit chronischer HCV-Infektion weisen eine höhere Inzidenz einer Niereninsuffizienz auf als Patienten ohne HCV-Infektion. Auch die Zeit bis zur Entwicklung der Niereninsuffizienz bzw. einer terminalen Niereninsuffizienz war bei den HCV-Patienten signifikant kürzer [5]. Die KDIGOLeitlinie empfiehlt, bei jeder medikamentösen Therapie auf die Nierenfunktion zu achten [3]. Potenziell nephrotoxische und renal eliminierte Medikamente sollten bei Patienten mit einer GFR $<60 \mathrm{ml} / \mathrm{min} / 1,73 \mathrm{~m}^{2}$ zeitweise abgesetzt werden, sobald eine schwere Erkrankung auftritt, die das Risiko für ein akutes Nierenversagen erhöht.

\section{Aktuelle HCV-Epidemiologie}

In Deutschland liegt die Prävalenz der chronischen Hepatitis $C$ bei etwa 0,3\% [13]. Bisher sind sechs Genotypen (GT1-6) des Virus bekannt. Mit 73,8\% steht der GT1 in Deutschland in der Häufigkeit an erster Stelle. Allein der Subgenotyp 1b macht $38,4 \%$ aller Erkrankungen aus. Der GT4 findet sich bei 4,2\% aller Erkrankten [14].

\section{Ein DAA-Regime auch für}

\section{Niereninsuffiziente}

Dies hat auch Implikationen für die antivirale Therapie der Hepatitis C. Diese ist durch Einführung direkt antiviral wirksamer Substanzen (direct-acting antiviral agents; DAAs) extrem effektiv geworden: Nahezu alle Patienten mit chronischer HCV-Infektion können heute mit DAA-Regimes geheilt werden. Für Patienten mit schwerer Nierenfunktionsstörung kommen jedoch nicht alle antiviralen Medikamente gleichermaßen in Betracht. Beispielsweise sollte laut Leitlinie der DGVS Sofosbuvir nicht bei Patienten mit schwerer Niereninsuffizienz $(<30 \mathrm{ml} /$ min eGFR) eingesetzt werden, da unter anderem die Metabolite renal eliminiert werden [2]. Das HCV-Therapieregime Ombitasvir/Paritaprevir/ Ritonavir (OBV/PTV/r; Viekirax) \pm Dasabuvir (DSV; Exviera) mit oder ohne Ribavirin (RBV) enthält 2 bzw. 3 DAAs (Ombitasvir, Paritaprevir, Dasabuvir), die im Wesentlichen alle hepatobiliär eliminiert werden [6, 7]. Die deutsche HCV-Leitlinie nennt dieses Regime als einzige zugelassene Therapieoption, die auch bei Patienten mit schwerer Niereninsuffizienz eingesetzt werden kann [2]. Eine Dosisanpassung ist nicht erforderlich.

\section{Sehr hohe Heilungsraten}

$\mathrm{OBV} / \mathrm{PTV} / \mathrm{r} \pm$ DSV ist seit dem 15. Januar 2015 in der EU zugelassen für Patienten mit chronischer HCV-Infektion vom Genotyp 1 (GT1) und GT4. Darin eingeschlossen sind auch Patienten mit einer Nierenfunktionsstörung $\mathrm{CrCl} \geq 15$ $\mathrm{ml} / \mathrm{min}$. In klinischen Phase-III-Studien erzielte OBV/PTV/r + DSV mit oder ohne RBV bei GT1-Patienten Heilungsraten (sustained virologic response; SVR12) von bis zu 100\% - bei guter Verträglichkeit und einer Behandlungsdauer von zumeist 12 Wochen $[6,7]$. In der Phase-IIIbStudie RUBY-I (Kohorte 1) wurde auch bei $90 \%$ $(\mathrm{n}=18 / 20)$ der nicht vorbehandelten, nicht zirrhotischen GT1-Patienten mit schwerer Niereninsuffizienz (CKD 4 und 5) einschließlich Dialyse-Patienten eine SVR12 erzielt [8]. In Kohorte 2 der RUBY-I waren auch vorbehandelte Patienten mit kompensierter Zirrhose (ChildPugh A) eingeschlossen. Eine SVR12 erzielten 89\% $(n=16 / 18)$ der Studienteilnehmer [9]. Patienten mit GT4-Infektion haben mit OBV/PTV/r mit oder ohne RBV über 12 oder 24 Wochen SVR12-Raten von $100 \%$ erreicht.

Daten aus dem Deutschen Hepatitis C-Register (DHC-R) bestätigen die hohe Wirksamkeit und gute Verträglichkeit von OBV/PTV/r \pm DSV mit oder ohne RBV auch unter Alltagsbedingungen: Eine SVR12 erreichten 96\% der GT1- und 100\% der GT4-Patienten. Von den Studienteilnehmern mit einer GFR $<60 \mathrm{ml} / \mathrm{min} / 1,73 \mathrm{~m}^{2}$ erzielten $100 \%$ eine SVR12 [10].

Auf Basis der Preisangaben in der Lauer-Taxe und der empfohlenen Therapieregime stellt dieses Regime die wirtschaftlichste interferonfreie 12-Wochen-Therapie dar [11]. Zudem erhielt die Kombinationstherapie die beste Nutzenbewertung des Gemeinsamen Bundesausschusses (G-BA) im Bereich Hepatitis C: Ein Zusatznutzen wurde für 99,5\% der zulassungsgemäßen Patientengruppen attestiert, darunter alle GT1-Patienten. Erstmals erteilte der G-BA zudem einen beträchtlichen Zusatznutzen für ein Interferonfreies Therapieregime - für therapienaive GT1-Patienten ohne Zirrhose [12].

\section{Fazit in Stichpunkten}

Die Nierenfunktion muss für die antivirale HCV-Therapie berücksichtigt werden.

Laut Leitlinie ist Ombitasvir/Paritaprevir/ Ritonavir \pm Dasabuvir mit oder ohne Ribavirin die derzeit einzige Therapieoption für Patienten mit schwerer Niereninsuffizienz. Eine Dosisanpassung ist nicht nötig [2]. Unter GT1-Patienten mit Nierenfunktionsstörung (CKD 4 und 5) erzielte die Kombinationstherapie in klinischen Studien Heilungsraten von 89 bis $90 \%$, darunter auch Dialyse-Patienten $[8,9]$.

Dr. Angelika Bischoff, Planegg

\section{Literatur}

1 Molnar MZ et al.: Hepatology 2015;61:1495-1502

2 Sarrazin C et al.: Z Gastroenterol 2015;53:320-334. Gültige Leitlinie der DGVS: Stand 10. August 2016. 3 KDIGO Guidelines CKD. Kidney Int Suppl 2013;3:5-14.

4 Brueck K et al.: J Am Soc Nephrol 2016;27:2135-2147.

5 Satapathy SK et al.: Hepatol Int 2012;6:369-378.

6 Fachinformation Viekirax ${ }^{\circledR}$; Stand April 2016.

7 Fachinformation Exviera ${ }^{\circledR}$; Stand April 2016.

8 Pockros PJ et al.: Gastroenterol 2016;150:1590-1598.

9 Hassanein T et al.: APASL, 12. Juni 2016.

10 Hinrichsen H et al.: J Hepatol 2016;64(suppl 2):S159, abstr GS07.

11 Lauer-Taxe, Apothekenverkaufspreise inkl. Mehrwertsteuer zum 1. August 2016.

12 G-BA. https://www.g-ba.de/informationen/beschluesse/2295, Stand: 10. August 2016.

3 Robert Koch-Institut. Epid Bull 2015;30:289-299. 14 Hüppe D et al.: J Viral Hepat 2015;22(suppl S3):22.

\section{Impressum}

Chronische Hepatitis C Nierenfunktion wichtig für die Therapieentscheidung

PharmaForum in VISCERAL Medicine 32 | 4 | 16

(c) 2016 by S. Karger Verlag für Medizin

und Naturwissenschaften GmbH Wilhelmstraße 20A, 79098 Freiburg, Deutschland

Mit freundlicher Unterstützung durch AbbVie Deutschland GmbH \& Co. KG

Verlag, Herausgeber, Redaktion und Verlagsgeschäftsführung übernehmen keine Verantwortung für den Inhalt dieser Rubrik. 